\title{
The Impact of Persistent Leukoencephalopathy on Brain White Matter Microstructure in Long-Term Survivors of Acute Lymphoblastic Leukemia Treated with Chemotherapy Only
}

(D) N.D. Sabin, DY.T. Cheung, (D)W.E. Reddick, (DD. Bhojwani, DW. Liu, DJ.O. Glass, (DT.M. Brinkman, DS.N. Hwang, (DD. Srivastava, (D) C.-H. Pui, DL.L. Robison, (D)M.M. Hudson, and DK.R. Krull O- E

\begin{abstract}
BACKGROUND AND PURPOSE: Survivors of acute lymphoblastic leukemia are at risk for neurocognitive deficits and leukoencephalopathy. We performed a longitudinal assessment of leukoencephalopathy and its associations with long-term brain microstructural white matter integrity and neurocognitive outcomes in survivors of childhood acute lymphoblastic leukemia treated on a modern chemotherapy-only protocol.
\end{abstract}

MATERIALS AND METHODS: One hundred seventy-three survivors of acute lymphoblastic leukemia (49\% female), treated on a chemotherapy-only protocol, underwent brain MR imaging during active therapy and repeat imaging and neurocognitive testing at follow-up (median, 13.5 years of age; interquartile range, 10.7-17.6 years; median time since diagnosis, 7.5 years; interquartile range, 6.3-9.1 years). Persistence of leukoencephalopathy was examined in relation to demographic and treatment data and to brain DTI in major fiber tracts and neurocognitive testing at follow-up.

RESULTS: Leukoencephalopathy was found in 52 of 173 long-term survivors (30.0\%) and persisted in 41 of 52 (78.8\%) who developed it during therapy. DTI parameters were associated with leukoencephalopathy in multiple brain regions, including the corona radiata (fractional anisotropy, $P=.001$; mean diffusivity, $P<.001$ ), superior longitudinal fasciculi (fractional anisotropy, $P=.02$; mean diffusivity, $P<$ .001 ), and superior fronto-occipital fasciculi (fractional anisotropy, $P=.006$; mean diffusivity, $P<.001$ ). Mean diffusivity was associated with neurocognitive impairment including in the genu of the corpus callosum $(P=.04)$, corona radiata $(P=.02)$, and superior frontooccipital fasciculi $(P=.02)$.

CONCLUSIONS: Leukoencephalopathy during active therapy and neurocognitive impairment at long-term follow-up are associated with microstructural white matter integrity. DTI may be more sensitive than standard MR imaging for detection of clinically consequential white matter abnormalities in childhood acute lymphoblastic leukemia survivors treated with chemotherapy and in children undergoing treatment.

ABBREVIATION: ALL = acute lymphoblastic leukemia

ontemporary treatment for pediatric acute lymphoblastic leukemia (ALL) has largely eliminated the use of prophylactic cranial radiation therapy ${ }^{1,2}$ and has substantially reduced many

Received March 26, 2018; accepted after revision July 19

From the Departments of Diagnostic Imaging (N.D.S., W.E.R., J.O.G., S.N.H.), Epidemiology and Cancer Control (Y.T.C., T.M.B., L.L.R., M.M.H., K.R.K.), Biostatistics (W.L., D.S.), Psychology (T.M.B., K.R.K.), and Oncology (C.-H.P., M.M.H.), St. Jude Children's Research Hospital, Memphis, Tennessee; and Children's Center for Cancer and Blood Diseases (D.B.), Children's Hospital Los Angeles, Los Angeles, California.

Noah D. Sabin and Yin Ting Cheung contributed equally to this work.

This work was supported by the National Institute of Mental Health Grant No. R01 MH085849, National Cancer Institute Grant No. R01 CA090246, and National Cancer Institute Cancer Center Support Grant No. P30 CA021765.

Paper previously presented, in part, at: Annual Meeting of the American Society of Clinical Oncology, May 29 to June 2, 2015; Chicago, Illinois.

Please address correspondence to Noah D. Sabin, MD, JD, Department of Diagnostic Imaging, St. Jude Children's Research Hospital, 262 Danny Thomas Place, MS

220, Memphis, TN 38105-3678; e-mail; noah.sabin@stjude.org adverse effects such as neurocognitive deficits, metabolic and endocrine disorders, and subsequent neoplasms. ${ }^{1,3,4}$ Parallel to this advance has been the use of risk-directed systemic and intrathecal chemotherapy, which has increased survival rates to $>90 \%$ in some studies. ${ }^{1}$ Although the neurocognitive function of survivors treated with chemotherapy only is better preserved than in those who underwent cranial radiation therapy, these survivors do demonstrate deficiencies. ${ }^{2,5}$

High doses of methotrexate, one of the primary chemotherapeutic agents used for consolidation treatment of ALL, are associated with leukoencephalopathy (ie, white matter hyperintensities on brain MR imaging). ${ }^{6,7}$ These changes may be transient or

\footnotetext{
- Indicates open access to non-subscribers at www.ajnr.org

$\equiv$ Indicates article with supplemental on-line tables.

Indicates article with supplemental on-line photo.

http://dx.doi.org/10.3174/ajnr.A5791
}

AJNR Am J Neuroradiol 39:1919-25 Oct 2018 www.ajnr.org 
persist over multiple MR imaging examinations conducted during and after chemotherapy. Leukoencephalopathy is sometimes associated with clinical findings of neurotoxicity such as strokelike symptoms, seizures, or aphasia. ${ }^{6,8}$

DTI is an MR imaging technique to evaluate microstructural white matter integrity based on the diffusion of water in the brain. Water diffusion in intact white matter is anisotropic (ie, diffusion is limited in direction, presumably due to cell membranes and myelin sheaths in parallel axonal fibers associated with white matter tracts). ${ }^{9}$ Fractional anisotropy is a measure of unidirectionality of water diffusion on a scale of $0-1$, with 0 representing no limitation in direction and 1 indicating diffusion completely confined to 1 direction. Mean diffusivity represents the average amount of diffusion regardless of direction. ${ }^{10-12}$ Injury to a white matter tract is associated with lower fractional anisotropy and higher mean diffusivity compared with healthy white matter. ${ }^{13,14}$

In a previous study of long-term survivors of childhood ALL treated with chemotherapy only, we reported associations between neurobehavioral outcomes and on-treatment leukoencephalopathy and DTI parameters in the frontostriatal tract, a tract we a priori predicted to be associated with executive function problems. ${ }^{15}$ We also examined pharmacologic determinants of neurocognitive performance, cortical thickness, and functional MR imaging in this cohort. ${ }^{16}$ In the current study, we more broadly examined the association between white matter integrity and neurocognitive outcomes in survivors by evaluating persistent leukoencephalopathy and DTI parameters in all major white matter tracts in the brain. We hypothesized that survivors who demonstrated persistent leukoencephalopathy would demonstrate poor microstructural white matter integrity as measured by DTI parameters and that poor white matter integrity would be associated with neurocognitive impairment.

\section{MATERIALS AND METHODS Study Participants}

Four hundred eight children with ALL were treated at St. Jude Children's Research Hospital on a single protocol (June 2000 to October 2010; Total Therapy XV, Total Therapy Study XV for Newly Diagnosed Patients with Acute Lymphoblastic Leukemia; clinicaltrials.gov No. NCT00137111) without prophylactic cranial radiation therapy. Of these, 369 underwent MR imaging examinations of the brain at 4 time points during therapy to assess development, progression, and/or resolution of leukoencephalopathy. ${ }^{6}$ Survivors were recruited during a long-term follow-up clinical evaluation when they were at least 8 years of age and $\geq 5$ years from diagnosis. Exclusion criteria included the following: relapse, diagnosis of a subsequent neoplasm, lack of proficiency in English, or the presence of an unrelated neurologic disorder associated with cognitive impairment. Among the 295 survivors who fulfilled the eligibility criteria, 189 (64\%) participated in long-term outcome studies between June 2009 and October 2014. Follow-up MR imaging of the brain was successfully performed for 173 of the survivors (On-line Figure). This study was approved by the institutional review board, and written informed consent was obtained from the patients, their parents, or guardians, as appropriate.

\section{Treatment}

The details of protocol therapy have been previously reported. ${ }^{1,17}$ Briefly, central nervous system-directed therapy comprised 13-18 triple intrathecal treatments with methotrexate, hydrocortisone, and cytarabine and 4 doses of high-dose intravenous methotrexate at an average of $2.5 \mathrm{~g} / \mathrm{m}^{2}$ per dose for patients with low-risk ALL. Patients with standard- or high-risk ALL received 16-25 triple intrathecal treatments and 4 doses of high-dose methotrexate at an average of $5.0 \mathrm{~g} / \mathrm{m}^{2}$ per dose. No patient received prophylactic central nervous system radiation therapy, even in the presence of central nervous system leukemia at diagnosis.

\section{MR Imaging}

MR imaging examinations for the follow-up study included noncontrast 3D sagittal T1WI, axial T2WI, and axial proton-density and axial T2 FLAIR images obtained on a 1.5T platform. DTI of the brain was successfully obtained on a $1.5 \mathrm{~T}$ platform using a double spin-echo EPI pulse sequence with 12 noncollinear, noncoplanar diffusion-gradient directions. Imaging sets were acquired with a spatial resolution of $1.7 \times 1.7 \times 3.0 \mathrm{~mm}$ and 4 acquisitions to ensure the highest signal-to-noise ratio possible within a limited amount of time. Voxelwise tensor calculations were performed with the Diffusion II toolkit under SPM8 (https://www.fil.ion.ucl.ac.uk/spm/). DTI measures of fractional anisotropy and mean diffusivity were extracted from voxels within fiber tracts throughout the brain.

All active therapy and long-term follow-up MR imaging examinations were reviewed for leukoencephalopathy by a boardcertified neuroradiologist with a Certificate of Added Qualification in neuroradiology and graded according to the radiographic criteria of the Common Terminology Criteria for Adverse Events (Version 4.0; https://www.eortc.be/services/doc/ctc/CTCAE_ 4.03_2010-06-14_QuickReference_5x7.pdf). The neuroradiologist was blinded to patient risk stratification, neurocognitive test performance, and DTI results. Hyperintensity on the T2weighted and T2 FLAIR images in the supratentorial white matter was considered leukoencephalopathy if located in the supraventricular white matter and/or periventricular white matter and more prominent than expected for terminal zones of myelination, normal developmental areas of T2 hyperintensity, for each subject's age. ${ }^{18}$ A second board-certified neuroradiologist with a Certificate of Added Qualification in neuroradiology separately graded 30 of the MR imaging examinations to estimate interrater reliability, yielding a $\kappa$ statistic of 0.75 (95\% CI, 0.64-0.85), indicating substantial agreement.

\section{Neurocognitive Testing}

Neurocognitive testing was performed by certified examiners under the supervision of a board-certified clinical neuropsychologist. Testing procedures followed standard clinical guidelines, with fixed test order and a schedule to reduce the effects of interference and fatigue. Testing evaluated executive function (cognitive flexibility, cognitive fluency, working memory, organization, and problem-solving abilities), ${ }^{19}$ intelligence (intelligence quotient), ${ }^{20}$ processing speed, ${ }^{21,22}$ attention, ${ }^{23}$ memory, ${ }^{24}$ and finemotor dexterity. ${ }^{25}$ Raw scores for these neurocognitive tests were 
Table 1: Demographics and treatment characteristics $(N=173)$

\begin{tabular}{|c|c|c|c|}
\hline & No. (\%) & Mean (SD) & Median (IQR) \\
\hline \multicolumn{4}{|l|}{ Demographics } \\
\hline \multicolumn{4}{|l|}{ Sex } \\
\hline Male & $89(51)$ & & \\
\hline Female & $84(49)$ & & \\
\hline \multicolumn{4}{|l|}{ Race/ethnicity } \\
\hline White & $124(72)$ & & \\
\hline Asian & $3(2)$ & & \\
\hline Black & $21(12)$ & & \\
\hline Hispanic & $18(10)$ & & \\
\hline Others & $7(4)$ & & \\
\hline Current age (yr) & & $14.4(4.6)$ & $13.5(10.7-17.6)$ \\
\hline Patient's highest education (yr) & & $7.7(3.9)$ & $7.0(4.0-11.0)$ \\
\hline Maternal education (yr) & & $13.6(2.5)$ & $13.0(12.0-16.0)$ \\
\hline Paternal education (yr) & & $13.6(3.1)$ & $12.0(12.0-16.0)$ \\
\hline \multicolumn{4}{|l|}{ Treatment characteristics } \\
\hline Age at diagnosis (yr) & & $6.7(4.3)$ & $5.3(3.5-8.6)$ \\
\hline Time since diagnosis (yr) & & $7.7(1.8)$ & $7.5(6.3-9.1)$ \\
\hline \multicolumn{4}{|l|}{ Treatment risk stratum } \\
\hline Low & $102(59)$ & & \\
\hline Standard & $71(41)$ & & \\
\hline \multicolumn{4}{|l|}{ Chemotherapy doses $^{\mathrm{a}}$} \\
\hline Oral dexamethasone $\left(\mathrm{mg} / \mathrm{m}^{2}\right)$ & & $1096.4(303.2)$ & $1099.9(985.3-1246.1)$ \\
\hline IV high-dose cytarabine $\left(\mathrm{g} / \mathrm{m}^{2}\right)$ & & $8.5(3.5)$ & $8.0(8.0-8.0)$ \\
\hline IV leucovorin $\left(\mathrm{mg} / \mathrm{m}^{2}\right)$ & & 343.5 (207.1) & $300.0(220.0-390.0)$ \\
\hline IV high-dose methotrexate $\left(\mathrm{g} / \mathrm{m}^{2}\right)$ & & $15.4(6.7)$ & $14.2(11.4-19.0)$ \\
\hline IT MHA (No. of counts) & & $14.4(4.0)$ & $13.0(12.0-16.0)$ \\
\hline
\end{tabular}

Note:- IQR indicates interquartile range; IT MHA, intrathecal injection of methotrexate plus hydrocortisone plus cytarabine.

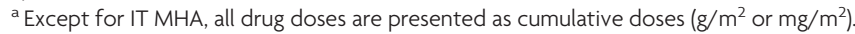

${ }^{\mathrm{b}}$ High-dose IV methotrexate was defined as a daily dose of $>1 \mathrm{~g} / \mathrm{m}^{2}$ of IV methotrexate.
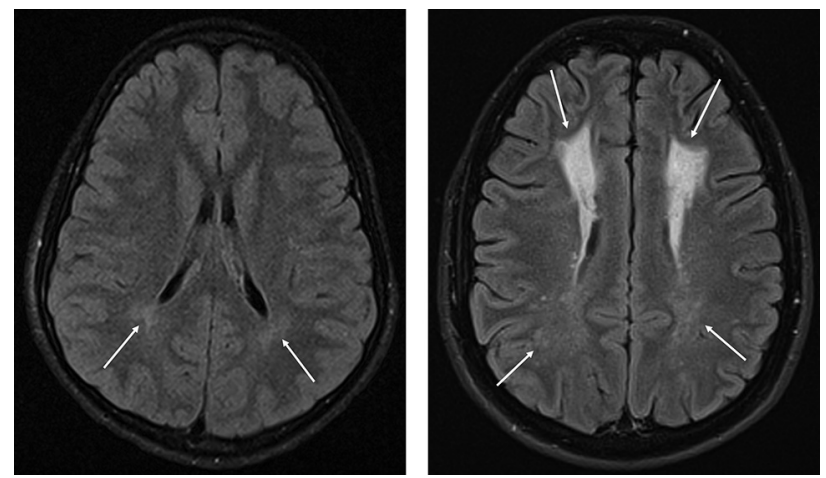

FIG 1. Examples of leukoencephalopathy grading. Left, mild hyperintensity in the bilateral periatrial white matter in this survivor of childhood ALL is compatible with grade 1 leukoencephalopathy according to the Common Terminology Criteria for Adverse Events (Version 4.0). Right, more extensive and confluent hyperintensity in the periventricular white matter that extends into the bilateral supraventricular frontoparietal white matter is considered grade 2 leukoencephalopathy.

transformed into age-adjusted $z$ scores based on nationally representative normative data $(\mu=0, \sigma=1.0)$. Applying our previous methods, ${ }^{16}$ we selected neurocognitive measures on which survivors' performance differed from normative samples. Survivors with $\geq 2$ neurocognitive test results $>1.5$ SDs or 1 test result $>2$ SDs below age-adjusted normative data were considered to have global neurocognitive impairment.

\section{Statistical Analysis}

For demographic and clinical data, means, SDs, medians, and interquartile ranges were calculated for continuous variables and frequencies were reported for categoric variables. The Mann-Whitney $U$ test (continuous variables) and the $\chi^{2}$ test (categoric variables) were used to evaluate whether demographic and clinical characteristics were different between survivors with and without persistent leukoencephalopathy. Associations between persistent leukoencephalopathy and white matter integrity (fractional anisotropy and mean diffusivity) were examined using general linear modeling, adjusting for current age. $P$ values were adjusted by controlling for the false discovery rate within the global white matter tracts. Only tracts that were significantly associated with leukoencephalopathy were tested for associations with methotrexate exposure using general linear modeling. Neurocognitive scores were transformed into age-adjusted $z$ scores ( $\mu=0, \sigma=1.0)$ using nationally representative norms. Onesample $t$ tests were applied to compare the group average with the expected population value $(\mu=0)$ for the specific tests. Impairment on an individual neurocognitive test was defined as a score falling below the tenth percentile of the norm $(z$ score $\leq-1.286)$. Global neurocognitive impairment was defined as having $\geq 2$ neurocognitive test scores that fall $>1.5$ SDs or 1 score that falls $>2$ SDs below the mean. Associations between neurocognitive scores and persistent leukoencephalopathy were evaluated using general linear modeling, adjusting for age at evaluation. Associations between global neurocognitive impairment and white matter integrity were assessed using general linear modeling, adjusted for age at evaluation. All analyses were conducted in SAS (SAS 9.4; SAS Institute, Cary, North Carolina). Statistical significance was defined as a $P$ value $<.05$, and all statistical tests were 2 -sided.

\section{RESULTS}

Demographic and treatment-related data are provided in Table 1, and their associations with leukoencephalopathy are shown in Online Table 1. Race/ethnicity for each subject was obtained from the medical record as self-reported by the patients' parents at the time of treatment. Of the 41 survivors with persistent leukoencephalopathy (leukoencephalopathy that was present on both active therapy and long-term follow-up MR imaging examinations), 37 had grade 1 and 4 had grade 2 according to the Common Terminology Criteria for Adverse Events (Version 4.0) (Fig 1). Because of the small number of participants with grade 2 abnormality, leukoencephalopathy was treated as a binary variable (present or absent). There were no significant differences in the cumulative dosages of known neurotoxic chemotherapeutic agents between patients with or without persistent leukoencephalopathy (On-line Table 1).

After correcting for the false discovery rate, there were significant associations between age-adjusted DTI parameters in mul- 


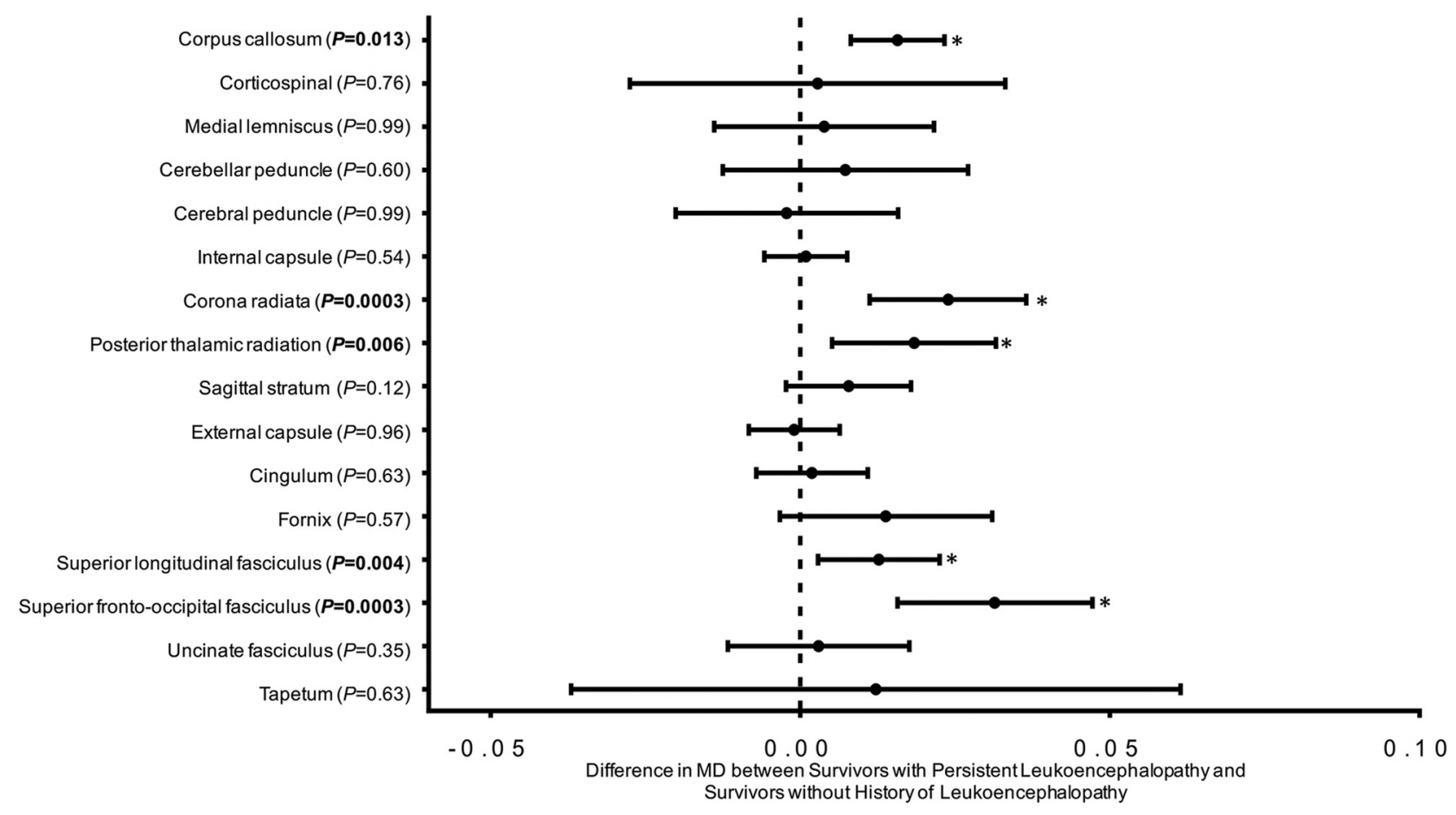

FIG 2. Leukoencephalopathy and white matter integrity. MD indicates mean diffusivity. Higher MD is indicative of worse white matter integrity. Point estimates represent the differences in white matter integrity between survivors with persistent leukoencephalopathy and survivors without a history of leukoencephalopathy within the global tracts listed on the x-axis. Error bars represent the $95 \%$ confidence intervals. The $P$ values compare the MD between survivors with and without acute leukoencephalopathy using general linear modeling, adjusted for current age. All models have been corrected for the false discovery rate within the global tracts. The image shows that survivors with persistent leukoencephalopathy had reduced white matter integrity, demonstrated by higher global MD, in the corpus callosum, corona radiata, posterior thalamic radiations, superior longitudinal fasciculi, and superior fronto-occipital fasciculi. Details on the associations between leukoencephalopathy and lateralized diffusion tensor imaging measures within the specific tracts can be found in On-line Table 2.

tiple fiber tracts and the presence of persistent leukoencephalopathy (Fig 2 and On-line Table 2). Lower fractional anisotropy and higher mean diffusivity were demonstrated in the corpus callosum, corona radiata, superior longitudinal fasciculi, and superior fronto-occipital fasciculi; higher mean diffusivity was detected in the posterior thalamic radiations in survivors with leukoencephalopathy. The total number of intrathecal administrations of methotrexate, hydrocortisone, and cytarabine was associated with higher mean diffusivity values in several fiber tracts (Table 2). High-dose intravenous methotrexate was not associated with DTI parameters.

Survivors had lower performance scores than population norms on measures of executive function, memory, processing speed, and global intelligence (almost all $P<.001$ ). There were no significant associations between neurocognitive performance measures and the presence of persistent leukoencephalopathy (On-line Table 3). However, higher mean diffusivity was associated with overall neurocognitive impairment (Fig 3 and On-line Table 4).

\section{DISCUSSION}

To our knowledge, this is the largest study of persistent leukoencephalopathy and tractography-based examination of white matter integrity in long-term survivors of childhood ALL treated with chemotherapy only. With serial imaging of the brain conducted during therapy and $>5$ years following diagnosis in 173 survivors, we identified the prevalence of persistent leukoencephalopathy to be $23.7 \%$, with $78.8 \%$ of survivors who developed acute leukoen- cephalopathy continuing to demonstrate leukoencephalopathy at long-term follow-up. Persistent leukoencephalopathy was associated with microstructural indices of poor white matter integrity on DTI, though only the DTI parameters were associated with clinically relevant neurocognitive outcomes. Such findings suggest that DTI may be a better method for evaluation of white matter pathology in patients and survivors of childhood ALL.

A study of 54 patients treated on 2 Pediatric Oncology Group ALL protocols reported a prevalence of leukoencephalopathy of $22 \%$ for one protocol and $68 \%$ for the second at roughly 7 years following diagnosis. ${ }^{26}$ The investigators attributed the higher prevalence in the second treatment group to the greater use of triple intrathecal therapy, increased frequency of intrathecal therapy, and lack of leucovorin rescue compared with the first group. The approximately $24 \%$ prevalence of leukoencephalopathy observed in the present investigation is similar to that reported in survivors in the first group of the Pediatric Oncology Group study. We detected no significant associations between specific chemotherapy cumulative doses and leukoencephalopathy.

The association between poor DTI parameters in multiple supratentorial white matter tracts and the presence of leukoencephalopathy in our survivors confirms the persistence of white matter injury well into long-term follow-up. While our DTI results are similar to those previously reported by others, ${ }^{13,27-33}$ our novel linkage of DTI to leukoencephalopathy suggests that white matter integrity is negatively impacted during the early course of chemotherapy treatment. 
Table 2: Association between methotrexate and white matter integrity ${ }^{\mathrm{a}}$

\begin{tabular}{|c|c|c|c|c|c|c|c|c|c|c|c|c|}
\hline \multirow[b]{3}{*}{ Tracts } & \multicolumn{6}{|c|}{ Intrathecal $\mathrm{MHA}^{\mathrm{b}}$} & \multicolumn{6}{|c|}{ High-Dose Methotrexate } \\
\hline & \multicolumn{3}{|c|}{ Mean Diffusivity } & \multicolumn{3}{|c|}{ Fractional Anisotropy } & \multicolumn{3}{|c|}{ Mean Diffusivity } & \multicolumn{3}{|c|}{ Fractional Anisotropy } \\
\hline & Est. & SE & $P$ & Est. & SE & $P$ & Est. & SE & $P$ & Est. & SE & $P$ \\
\hline Corpus callosum & 0.0017 & 0.0008 & $.04^{\mathrm{c}}$ & -0.0009 & 0.0007 & .21 & -0.0003 & 0.0005 & .60 & 0.0006 & 0.0004 & .17 \\
\hline Genu & 0.0016 & 0.0009 & .07 & -0.0015 & 0.0012 & .21 & -0.0001 & 0.0005 & .82 & -0.0002 & 0.0007 & .76 \\
\hline Body & 0.0008 & 0.0010 & .45 & 0.0000 & 0.0009 & .99 & -0.0008 & 0.0006 & .21 & 0.0011 & 0.0005 & .05 \\
\hline Splenium & 0.0022 & 0.0011 & $.04^{\mathrm{c}}$ & -0.0011 & 0.0007 & .13 & 0.0000 & 0.0006 & .95 & 0.0007 & 0.0004 & .09 \\
\hline Corona radiata & 0.0014 & 0.0007 & $.04^{\mathrm{c}}$ & -0.0004 & 0.0005 & .36 & -0.0003 & 0.0004 & .44 & 0.0002 & 0.0003 & .57 \\
\hline Anterior (left) & 0.0017 & 0.0009 & .06 & -0.0010 & 0.0005 & .06 & -0.0002 & 0.0005 & .73 & -0.0001 & 0.0003 & .75 \\
\hline Anterior (right) & 0.0011 & 0.0009 & .19 & -0.0005 & 0.0006 & .41 & -0.0005 & 0.0005 & .31 & 0.0001 & 0.0003 & .78 \\
\hline Superior (left) & 0.0013 & 0.0005 & $.02^{c}$ & 0.0003 & 0.0007 & .70 & -0.0001 & 0.0003 & .69 & 0.0004 & 0.0004 & .33 \\
\hline Superior (right) & 0.0015 & 0.0005 & $.005^{c}$ & -0.0003 & 0.0007 & .66 & -0.0002 & 0.0003 & .58 & 0.0002 & 0.0004 & .53 \\
\hline Posterior (left) & 0.0009 & 0.0007 & .23 & 0.0003 & 0.0007 & .65 & -0.0007 & 0.0004 & .08 & 0.0007 & 0.0004 & .12 \\
\hline Posterior (right) & 0.0016 & 0.0007 & $.02^{c}$ & -0.0002 & 0.0007 & .79 & -0.0003 & 0.0004 & .42 & 0.0002 & 0.0004 & .58 \\
\hline Posterior thalamic radiation & 0.0015 & 0.0007 & $.03^{\mathrm{c}}$ & -0.0003 & 0.0007 & .63 & 0.0000 & 0.0004 & .94 & 0.0007 & 0.0004 & .11 \\
\hline Left & 0.0011 & 0.0010 & .29 & 0.0000 & 0.0008 & .98 & -0.0003 & 0.0006 & .65 & 0.0009 & 0.0005 & .05 \\
\hline Right & 0.0018 & 0.0006 & $.003^{c}$ & -0.0006 & 0.0007 & .42 & 0.0001 & 0.0004 & .69 & 0.0005 & 0.0004 & .24 \\
\hline $\begin{array}{l}\text { Superior longitudinal } \\
\text { fasciculus }\end{array}$ & 0.0007 & 0.0005 & .16 & 0.0006 & 0.0005 & .25 & -0.0001 & 0.0003 & .75 & 0.0005 & 0.0003 & .14 \\
\hline Left & 0.0006 & 0.0005 & .22 & 0.0008 & 0.0006 & .16 & -0.0001 & 0.0003 & .61 & 0.0004 & 0.0003 & .30 \\
\hline Right & 0.0007 & 0.0005 & .13 & 0.0005 & 0.0006 & .41 & 0.0000 & 0.0003 & .87 & 0.0006 & 0.0003 & .10 \\
\hline $\begin{array}{l}\text { Superior fronto-occipital } \\
\text { fasciculus }\end{array}$ & 0.0020 & 0.0009 & $.02^{c}$ & -0.0004 & 0.0009 & .65 & -0.0003 & 0.0005 & .60 & 0.0009 & 0.0005 & .09 \\
\hline Left & 0.0021 & 0.0010 & $.04^{c}$ & -0.0004 & 0.0009 & .63 & -0.0005 & 0.0006 & .41 & 0.0009 & 0.0005 & .10 \\
\hline Right & 0.0019 & 0.0007 & $.01^{\mathrm{c}}$ & -0.0003 & 0.0010 & .73 & 0.0000 & 0.0005 & .97 & 0.0008 & 0.0006 & .14 \\
\hline
\end{tabular}

Note:-Est. indicates parameter estimate; SE, standard error; MHA, methotrexate plus hydrocortisone plus cytarabine.

${ }^{a}$ General linear modeling was applied for the test of strength of association between treatment variables with mean diffusivity and fractional anisotropy for each tract, adjusted for age at evaluation. Higher mean diffusivity and lower fractional anisotropy are indicative of worse white matter integrity.

${ }^{b}$ Intrathecal MHA was defined as the number of intrathecal injections of methotrexate plus hydrocortisone plus cytarabine; high-dose IV methotrexate was defined as a daily dose of $>1 \mathrm{~g} / \mathrm{m}^{2}$, presented as cumulative doses $\left(\mathrm{g} / \mathrm{m}^{2}\right)$.

'Significant.

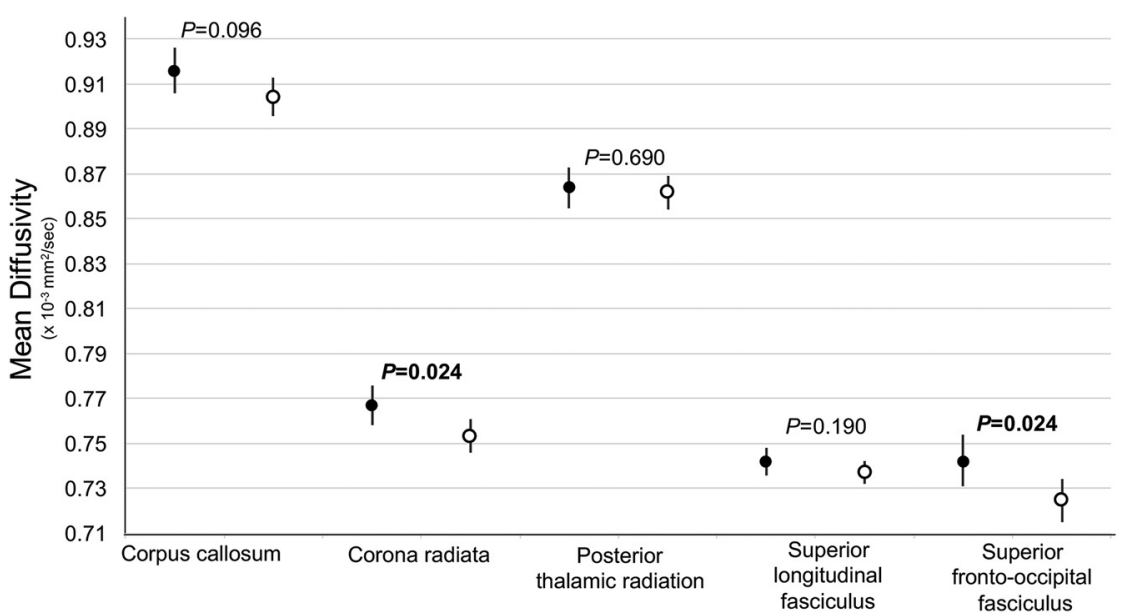

FIG 3. Association between white matter integrity and global neurocognitive impairment. Black circles represent survivors with global neurocognitive impairment. White circles represent survivors without global neurocognitive impairment. Global neurocognitive impairment is defined as having $\geq 2$ neurocognitive tests (listed in On-line Table 3 ) that fall $>1.5$ SDs or 1 test that falls $>2$ SDs below the age-adjusted population normative data. The $y$-axis represents mean diffusivity. Higher mean diffusivity is indicative of worse white matter integrity. Error bars represent the $95 \%$ confidence intervals. $P$ values compare the mean diffusivity between survivors with neurocognitive impairment (closed circle, $n=63$ ) and without neurocognitive impairment (open circle, $n=$ 87) using general linear modeling, adjusted for current age. The image shows that survivors with neurocognitive impairment had reduced white matter integrity demonstrated by higher global mean diffusivity in the corona radiata and superior fronto-occipital fasciculus. Details on the associations between global neurocognitive impairment and lateralized diffusion tensor imaging measures within the specific tracts can be found in On-line Table 4.

Despite associations with lower fractional anisotropy and higher mean diffusivity, leukoencephalopathy was not significantly associated with neurocognitive test performance. Duffner et $\mathrm{al}^{26}$ reported a significant association between leukoencephalopathy and poorer performance on tests of attention in a group of 43 ALL survivors treated with chemotherapy only. Although there were no significant differences in the mean full-scale intelligence quotient, verbal intelligence quotient, or performance intelligence quotient between patients with or without leukoencephalopathy, survivors with leukoencephalopathy composed $80 \%$ of those scoring $>1$ SD below the mean for verbal intelligence quotient; 64\%, for performance intelligence quotient; and $89 \%$, for full-scale intelligence quotient. ${ }^{26}$ In contrast, we did not see similar trends in $>150$ survivors who completed both MR imaging examinations and neurocognitive testing. However, our DTI measures were associated with neurocognitive function.

Mean diffusivity in the genu of the corpus callosum, corona radiata, and the superior fronto-occipital fasciculi was associated with global neurocognitive impairment. These tracts all involve the frontal lobes, which play a role in executive function ${ }^{16}$ and which demonstrate prolonged development during childhood, potentially placing them 
at extended risk for neurotoxicity. Other investigators have also found associations between abnormal DTI parameters and deficits in neurocognitive performance in survivors of ALL treated with chemotherapy only. ${ }^{13,27,28,32}$ That only mean diffusivity and not fractional anisotropy was associated with neurocognitive deficits suggests that the amount of diffusion, rather than the degree of anisotropy, in our survivors' white matter had a greater effect on their neurocognitive abilities. The larger number of associations between mean diffusivity in multiple fiber tracts and the total number of intrathecal administrations of chemotherapeutic agents, compared with fractional anisotropy, also indicates that chemotherapy has a greater effect on the amount of diffusion in those tracts than the degree to which it is anisotropic. This is an area that requires further investigation.

Limitations to our study include distinguishing leukoencephalopathy from terminal zones of myelination, which can be difficult in some cases. The grades of leukoencephalopathy were compared over multiple MR imaging examinations to try to standardize the visual thresholds used across the studies; however, it is impossible, on the basis of visual inspection alone, to be certain that some cases of leukoencephalopathy were not classified as terminal zones or that terminal zones may have been incorrectly considered leukoencephalopathy. Given the size of our cohort, though, the instances of misclassification likely did not have a large effect on our results. In addition, the strong correlation between the presence of leukoencephalopathy and DTI parameters suggests that misclassification did not significantly affect our data. Another limitation is that this investigation lacked a control group for comparison with the DTI findings. The differences in the DTI parameters between survivors with and without leukoencephalopathy and the linkage of the DTI measures to standardized neurocognitive testing, however, demonstrate the importance of our DTI results. The limited research DTI sequence used in this study was designed and implemented $>8$ years ago and was not optimal for tracking all possible connections. However, the connections reported were reproducible and reliable. Future studies will likely include higher spatial and angular resolution acquisitions. We had no pretreatment DTI of the brain, which prevented us from determining whether DTI parameters were already abnormal due to disease. Because treatment typically begins within 24 hours of diagnosis for ALL and because the imaging in this study was acquired solely for research, we were not able to obtain pretreatment imaging. Last, no neurocognitive testing was performed before active therapy; thus, some neurocognitive deficits in our subjects may have existed before treatment. The lack of a baseline study, however, is not likely to account for most of the problems in neurocognitive performance we detected.

Children successfully treated for ALL with chemotherapy alone are at risk for persistent leukoencephalopathy. Although leukoencephalopathy is an MR imaging biomarker of white matter abnormality, it was not associated with neurocognitive deficits in our survivors. Differences in DTI parameters, a more precise measure of microstructural white matter integrity, however, were associated with global neurocognitive impairment, suggesting that DTI may serve as a more sensitive imaging measure of clinically consequential loss of white matter integrity in ALL survivors than the presence of leukoencephalopathy on standard MR imag- ing sequences. While the precise significance of the loss of structural integrity that leukoencephalopathy represents is unclear, continued refinement of chemotherapy treatment regimens for ALL should nevertheless aim to reduce the occurrence of white matter abnormalities. Potential treatments and interventions to enhance white matter development and function also need to be explored.

\section{CONCLUSIONS}

Leukoencephalopathy that develops in patients treated for childhood ALL and long-term neurocognitive impairment are associated with microstructural white matter integrity. DTI may be more sensitive than standard MR imaging sequences for identifying clinically consequential white matter abnormalities in this group of cancer survivors.

\section{ACKNOWLEDGMENTS}

We would like to acknowledge Cara Kimberg, PhD, of Psychological Care Associates, Woburn, Massachusetts; and Ms Cynthia Jones, Ms Deborah Stewart, and Ms Adrienne Studaway of the Department of Epidemiology and Cancer Control, St. Jude Children's Research Hospital, for administering the neurocognitive tests; and Ms Joycelynn Butler, of the Department of Epidemiology and Cancer Control, St. Jude Children's Research Hospital, for extracting and cleaning the data.

Disclosures: Noah D. Sabin—RELATED: Grant: National Cancer Institute, National Institute of Mental Health.* Ching-Hon Pui-RELATED: Grant: National Institutes of Health CA 21765*. Leslie L. Robison—RELATED: Grant: National Institutes of Health*. Melissa M. Hudson—RELATED: Grant: National Cancer Institute, Comments: U01 195547*. Kevin R. Krull—RELATED: Grant: National Institute of Mental Health, National Cancer Institute*; UNRELATED: Grants/Grants Pending: National Cancer Institute*; Royalties: Wolters Kluwer Publishers, Comments: royalties for chapters written on attention-deficit/hyperactivity disorder for UpToDate. Chapters are unrelated to content of current work. *Money paid to the institution.

\section{REFERENCES}

1. Pui CH, Campana D, Pei D, et al. Treating childhood acute lymphoblastic leukemia without cranial irradiation. N Engl J Med 2009;360: 2730-41 CrossRef Medline

2. Cheung YT, Krull KR. Neurocognitive outcomes in long-term survivors of childhood acute lymphoblastic leukemia treated on contemporary treatment protocols: a systematic review. Neurosci Biobehav Rev 2015;53:108-20 CrossRef Medline

3. Hudson MM, Ness KK, Gurney JG, et al. Clinical ascertainment of health outcomes among adults treated for childhood cancer. JAMA 2013;309:2371-81 CrossRef Medline

4. Sabin ND, Santucci AK, Klimo P Jr, et al. Incidental detection of late subsequent intracranial neoplasms with magnetic resonance imaging among adult survivors of childhood cancer. J Cancer Surviv 2014;8:329-35 CrossRef Medline

5. Krull KR, Brinkman TM, Li C, et al. Neurocognitive outcomes decades after treatment for childhood acute lymphoblastic leukemia: a report from the St Jude Lifetime Cohort study. J Clin Oncol 2013; 31:4407-15 CrossRef Medline

6. Bhojwani D, Sabin ND, Pei D, et al. Methotrexate-induced neurotoxicity and leukoencephalopathy in childhood acute lymphoblastic leukemia. J Clin Oncol 2014;32:949-59 CrossRef Medline

7. Reddick WE, Glass JO, Helton KJ, et al. Prevalence of leukoencephalopathy in children treated for acute lymphoblastic leukemia with high-dose methotrexate. AJNR Am J Neuroradiol 2005;26:1263-69 Medline

8. Fisher MJ, Khademian ZP, Simon EM, et al. Diffusion-weighted MR 
imaging of early methotrexate-related neurotoxicity in children. AJNR Am J Neuroradiol 2005;26:1686-89 Medline

9. Choudhri AF, Chin EM, Blitz AM, et al. Diffusion tensor imaging of cerebral white matter: technique, anatomy, and pathologic patterns. Radiol Clin North Am 2014;52:413-25 CrossRef Medline

10. Raffin E, Dyrby TB. Diagnostic approach to functional recovery: diffusion-weighted imaging and tractography. Front Neurol Neurosci 2013;32:26-35 CrossRef Medline

11. Yang E, Nucifora PG, Melhem ER. Diffusion MR imaging: basic principles. Neuroimaging Clin N Am 2011;21:1-25, vii CrossRef Medline

12. Roberts RM, Mathias JL, Rose SE. Relationship between diffusion tensor imaging (DTI) findings and cognition following pediatric TBI: a meta-analytic review. Dev Neuropsychol 2016;41:176-200 CrossRef Medline

13. Edelmann MN, Krull KR, Liu W, et al. Diffusion tensor imaging and neurocognition in survivors of childhood acute lymphoblastic leukaemia. Brain 2014;137(Pt 11):2973-83 CrossRef Medline

14. Sidaros A, Engberg AW, Sidaros K, et al. Diffusion tensor imaging during recovery from severe traumatic brain injury and relation to clinical outcome: a longitudinal study. Brain 2008;131(Pt 2):559-72 CrossRef Medline

15. Cheung YT, Sabin ND, Reddick WE, et al. Leukoencephalopathy and long-term neurobehavioural, neurocognitive, and brain imaging outcomes in survivors of childhood acute lymphoblastic leukaemia treated with chemotherapy: a longitudinal analysis. Lancet Haematol 2016;3:e456-66 CrossRef Medline

16. Krull KR, Cheung YT, Liu W, et al. Chemotherapy pharmacodynamics and neuroimaging and neurocognitive outcomes in longterm survivors of childhood acute lymphoblastic leukemia. J Clin Oncol 2016;34:2644-53 CrossRef Medline

17. Pui CH, Relling MV, Sandlund JT, et al. Rationale and design of Total Therapy Study XV for newly diagnosed childhood acute lymphoblastic leukemia. Ann Hematol 2004;83(Suppl 1):S124-26 Medline

18. Welker KM, Patton A. Assessment of normal myelination with magnetic resonance imaging. Semin Neurol 2012;32:15-28 CrossRef Medline

19. Delis DC, Kaplan E, Kramer JH. Delis-Kaplan Executive Function System. San Antonio: Psychological Corporation; 2001

20. Wechsler D. Wechsler Abbreviated Scale of Intelligence. San Antonio: Psychological Corporation; 1999

21. Wechsler D. Wechsler Intelligence Scale for Children. 4th ed. San Antonio: Harcourt Assessment; 2003
22. Wechsler D. Wechsler Adult Intelligence Scale. 4th ed. San Antonio: Pearson; 2008

23. Conners CK. Conners' Continuous Performance Test II. North Tonawanda: Multi-Health Systems; 2001

24. Meyers JE, Meyers KR. Rey Complex Figure and Recognition Trial. Odessa: Psychological Assessment Resources; 1996

25. Strauss E, Sherman EM, Spreen O. A Compendium of Neuropsychological Tests: Administration, Norms, and Commentary. 3rd ed. New York: Oxford University Press; 2006

26. Duffner PK, Armstrong FD, Chen L, et al. Neurocognitive and neuroradiologic central nervous system late effects in children treated on Pediatric Oncology Group (POG) P9605 (standard risk) and P9201 (lesser risk) acute lymphoblastic leukemia protocols (ACCL0131): a methotrexate consequence? A report from the Children's Oncology Group. J Pediatr Hematol Oncol 2014;36:8-15 CrossRef Medline

27. Aukema EJ, Caan MW, Oudhuis N, et al. White matter fractional anisotropy correlates with speed of processing and motor speed in young childhood cancer survivors. Int J Radiat Oncol Biol Phys 2009; 74:837-43 CrossRef Medline

28. ElAlfy M, Ragab I, Azab I, et al. Neurocognitive outcome and white matter anisotropy in childhood acute lymphoblastic leukemia survivors treated with different protocols. Pediatr Hematol Oncol 2014; 31:194-204 CrossRef Medline

29. Genschaft M, Huebner T, Plessow F, et al. Impact of chemotherapy for childhood leukemia on brain morphology and function. PLoS One 2013;8:e78599 CrossRef Medline

30. Khong PL, Leung LH, Fung AS, et al. White matter anisotropy in post-treatment childhood cancer survivors: preliminary evidence of association with neurocognitive function. J Clin Oncol 2006;24: 884-90 CrossRef Medline

31. Porto L, Preibisch C, Hattingen E, et al. Voxel-based morphometry and diffusion-tensor MR imaging of the brain in long-term survivors of childhood leukemia. Eur Radiol 2008;18:2691-700 CrossRef Medline

32. Schuitema I, Deprez S, Van Hecke W, et al. Accelerated aging, decreased white matter integrity, and associated neuropsychological dysfunction 25 years after pediatric lymphoid malignancies. J Clin Oncol 2013;31:3378-88 CrossRef Medline

33. Zou L, Su L, Xu J, et al. Structural brain alteration in survivors of acute lymphoblastic leukemia with chemotherapy treatment: a voxel-based morphometry and diffusion tensor imaging study. Brain Res 2017;1658:68-72 CrossRef Medline 